\title{
A FORMAL EXPANSION THEORY FOR FUNCTIONS OF ONE OR MORE VARIABLES ${ }^{1}$
}

\author{
NEWMAN A. HALL
}

It is a familiar property of the expansion of a function in series of functions that the coefficients of ten may be expressed in terms of the coefficients of the Taylor series for the original function. This has been done explicitly for many specific cases with functions of a single variable. ${ }^{2}$ In this paper there is presented a method of obtaining more general results of this nature for functions of any number of variables defined by power series.

The umbral calculus introduced by Blissard in his Theory of generic functions $^{3}$ has been used by Lucas and Bell among others as a convenient instrument in the manipulation of generating functions. The algebra of the umbrae has been discussed by Bell ${ }^{4}$ and some of the simplest properties of these will be used in the theory presented below.

A function $f(x)$ defined by a power series ${ }^{5}$

$$
f(x)=\sum_{n} a_{n} \frac{x^{n}}{n !}
$$

may be equally well defined by the matrix

$$
a=\left|a_{0}, a_{1}, \cdots, a_{n}, \cdots\right| \text {. }
$$

The umbral calculus admits the equality $a^{n}=a_{n}$, that is, the $n$th power of the matrix is equal to the $n$th term. From this it follows that $f(x)=e^{a x}$.

Functions of several variables suggest a similar notation. The function

${ }^{1}$ Presented to the Society, November 27, 1937, under the title $A$ formal expansion theory for functions defined by two variable power series.

${ }^{2}$ N. Nielsen, Fonctions Métasphériques, chap. 4. N. Nielsen, Recherches sur le développement d'une fonction analytique en series de fonctions hypergéométriques, Annales Scientifiques d'École Normale Supérieure, (3), vol. 30 (1913), p. 12. S. Pincherle, Alcuni teoremi sopra gli sviluppi en serie per funzioni analitiche, Rendiconti dell' Istituto Lombardo di Scienze e Lettere, (2), vol. 15 (1882), p. 224. J. M. Whittaker, Interpolatory Function Theory, Cambridge, 1937.

${ }^{3}$ John Blissard, Quarterly Journal of Mathematics, vols. 4-6 (1861-1864).

${ }^{4}$ E. T. Bell, Algebraic Arithmetic, American Mathematical Society Colloquium Pubications, vol. 7, New York, 1927, pp. 146-159.

${ }^{5}$ All summations are to extend from 0 to $\infty$. In place of a repeated summation, $\sum_{n_{1}=0}^{\infty} \sum_{n_{2}=0}^{\infty} \cdots \sum_{n_{k}=0}^{\infty} A_{n_{1}, n_{2}}, \cdots, n_{k}$ we shall write $\sum_{n_{1}, \cdots, n_{k}} A_{n_{1}}, \cdots, n_{k}$. 


$$
f(x)=f\left(x_{1}, \cdots, x_{k}\right)=\sum_{n_{1}, \cdots, n_{k}} a_{n_{1}, \cdots, n_{k}} \frac{x_{1}^{n_{1}} \cdots x_{k}^{n_{k}}}{n_{1} ! \cdots n_{k} !}
$$

is defined also by the $k$-dimensional matrix

$$
a=a^{\prime} a^{\prime \prime} \cdots a^{(k)}=\left|a_{i_{1}, \ldots, i_{k}}\right| .
$$

The exponential property of the one-dimensional matrices may be extended by setting $a^{\prime n_{1}} a^{\prime \prime n_{2}} \cdots a^{(k) n_{k}}=a_{n_{1}, n_{2}}, \cdots, n_{k}$ so that

$$
f(x)=\exp \left\{a^{\prime} x_{1}+a^{\prime \prime} x_{2}+\cdots+a^{(k)} x_{k}\right\} .
$$

This use of an umbral notation puts the primary emphasis on the number of the term in the series and on the coefficient rather than on the variable.

We shall call two sets of functions

$$
P_{n}(x)=P_{n_{1}, \cdots, n_{k}}\left(x_{1}, \cdots, x_{k}\right), \quad Q_{n}(y)=Q_{n_{1}, \cdots, n_{k}}\left(y_{1}, \cdots, y_{k}\right)
$$

associate, if they satisfy the relation

$$
\exp \left\{x_{1} y_{1}+x_{2} y_{2}+\cdots+x_{k} y_{k}\right\}=\sum_{n_{1}, \cdots, n_{k}} \frac{P_{n}(x) Q_{n}(y)}{n_{1} ! \cdots n_{k} !} .
$$

In this discussion $P_{n}(x)$ and $Q_{n}(y)$ shall be restricted to functions defined by power series or by the matrices

$$
p_{n}=\left|p_{n_{1}}, \cdots, n_{k}: i_{1}, \cdots, i_{k}\right|, \quad q_{n}=\left|q_{n_{1}}, \cdots, n_{k} ; i_{1}, \cdots, i_{k}\right| .
$$

According to the theorem below, in order to expand $f(x)$ in a series of functions $P_{n}(x)$, it is only necessary to know the associate function $Q_{n}(y)$.

Theorem I. If $P_{n}(x)$ and $Q_{n}(y)$ are associate functions and $f(x)$ is a function defined by the matrix $a^{\prime} a^{\prime \prime} \cdots a^{(k)}$, the expansion of $f(x)$ in the series of functions $P_{n}(x)$ is

$$
\begin{aligned}
f(x) & =\exp \left\{a^{\prime} x_{1}+\cdots+a^{(k)} x_{k}\right\} \\
& =\sum_{n_{1}, \cdots, n_{k}} \frac{P_{n_{1}, \cdots, n_{k}}\left(x_{1}, \cdots, x_{k}\right) Q_{n_{1}}, \cdots, n_{k}\left(a^{\prime}, \cdots, a^{(k)}\right)}{n_{1} ! \cdots n_{k} !} .
\end{aligned}
$$

A discussion for functions of two variables will illustrate the argument required in general. From the definition of the associate functions,

or

$$
e^{x_{1} y_{1}+x_{2} y_{2}}=\sum_{m_{1}, m_{2}} \frac{P_{m_{1}, m_{2}}\left(x_{1}, x_{2}\right) Q_{m_{1}, m_{2}}\left(y_{1}, y_{2}\right)}{m_{1} ! m_{2} !}
$$




$$
\begin{gathered}
\sum_{n_{1}, n_{2}} \frac{x_{1}^{n_{1}} y_{1}^{n_{1}} x_{2}^{n_{2}} y_{2}^{n_{2}}}{n_{1} ! n_{2} !}=\sum_{m_{1}, m_{2}} \frac{1}{m_{1} ! m_{2} !} \sum_{r_{1}, r_{2}} \frac{p_{m_{1}, m_{2}: r_{1}, r_{2}}}{r_{1} ! r_{2} !} x_{1}^{r_{1}} x_{2}^{r_{2}} \\
\cdot \sum_{s_{1}, s_{2}} \frac{q_{m_{1}, m_{2}: s_{1}, s_{2}}}{s_{1} ! s_{2} !} y_{1}^{s_{1}} y_{2}^{s_{2}} .
\end{gathered}
$$

If $y_{1}^{h} y_{2}^{k}$ is replaced throughout by $a_{h, k}$, the equality exhibited is not affected in any way, since the variables in effect serve only to number the terms in the series. The equality follows from relations between the coefficients and is not dependent upon the variables. With this substitution made the theorem follows.

When one set of associate functions is known, any number of others may be derived by the use of the following result.

THEOREM II. If $P_{n}(x)$ and $Q_{n}(y)$ are a set of associate functions and $c^{\prime} c^{\prime \prime} \cdots c^{(k)}=c_{n_{1}}, \cdots, n_{k}$ is an arbitrary matrix, then

$$
P_{n}\left(x_{1} c^{\prime}, x_{2} c^{\prime \prime}, \cdots, x_{k} c^{(k)}\right), \quad Q_{n}\left(y_{1} / c^{\prime}, y_{2} / c^{\prime \prime}, \cdots, y_{k} / c^{(k)}\right)
$$

are associate functions.

Again the proof for two variables follows from an examination of the defining identity as given in the discussion of Theorem I. It is only necessary to replace $x_{1}^{r_{1}} x_{2}^{r_{2}}$ by $x_{1}^{r_{1}} x_{2}^{r_{2}} c_{r_{1}, r_{2}}$ and $y_{1}^{s_{1}} y_{2}^{s_{2}}$ by $y_{1}^{s_{1}} y_{2}^{s_{2}} / c_{s_{1}, s_{2}}$, which by reasoning as above will not affect the equality nor will it affect the left side since $c_{n_{1}, n_{2}}$ will cancel $1 / c_{n_{1}, n_{2}}$.

The associate functions may be obtained directly from the relation between the coefficients which follow from the identity defining the associate functions or more conveniently by applying Theorem II to certain basic sets. In the illustrations below it will be shown that a great number of expansions in functions of one or two variables will follow in this latter manner from two basic sets.

The Hermite polynomials suggest the more general class of functions $H_{n}^{p}(x)$, defined by the generating function

so that

$$
e^{z x-z^{p} / p}=\sum_{n} \frac{z^{n}}{n !} H_{n}^{p}(x),
$$

$$
H_{n}^{p}(x)=\sum_{s}(-)^{s(p+1)} \frac{(-n, p s)}{(1, s)} \frac{x^{n-p s}}{p^{s}} .
$$

The associate function $N_{n}^{p}(y)$ is readily determined from the generating function, since

$$
e^{x y}=e^{x y-y^{p} / p+y^{p} / p}=\sum_{n} \frac{H_{n}^{p}(x) y^{n} e^{y^{p} / p}}{n !},
$$


so that

$$
N_{n}^{p}(y)=y^{n} e^{y^{p} / p}=\sum_{r} \frac{y^{n+p r}}{p^{r}(1, r)} .
$$

The well known Neumann expansions in Bessel functions are special cases of the expansions obtained from the associate functions

$$
\begin{aligned}
& B_{n}^{k}(\alpha, x)=\sum_{r} \frac{x^{n+k r}}{(\alpha+2 n / k+1, r) r !}, \\
& L_{n}^{k}(\alpha, y)=\sum_{s}(-)^{k s} \frac{(-n, k s) y^{n-k s}}{(-\alpha-2 n / k+1, s) s !} .
\end{aligned}
$$

We show that these are associate by referring to the basic definition

$$
\begin{aligned}
\sum_{n} & \frac{B_{n}^{k}(\alpha, x) L_{n}^{k}(\alpha, y)}{n !} \\
= & \sum_{n, r, s}(-)^{k s} \frac{(-n, k s) x^{n+k r} y^{n-k s}}{(\alpha+2 n / k+1, r)(-\alpha-2 n / k+1, s) n ! r ! s !} \\
= & \sum_{p} \frac{x^{p} y^{p}}{p !} \\
& \cdot \sum_{r, s}(-)^{k(r+s)} \frac{(-p, k r)(-p+k r, k s) y^{-k r-k s}}{(-\alpha-2 p / k+2 r+1, s)(\alpha+2 p / k-2 r+1, r) r ! s !} \\
= & \sum_{p, q} \frac{x^{p} y^{p-k q}}{p ! q !} \\
& \cdot \sum_{r}(-)^{k r} \frac{(-q, r)(-p, k r)(-p+k r, k q-k r)}{(-\alpha-2 p / k+2 r+1, q-r)(\alpha+2 p / k-2 r+1, r) r !} \\
= & \sum_{p, q}(-)^{k q} \frac{(-p, k q) x^{p} y^{p-k q}}{(-\alpha-2 p / k+1, q) p ! q !} \\
& \cdot \sum_{r} \frac{(-q, r)(-\alpha-2 p / k+1,2 r)(-\alpha-2 p / k, r)}{(-\alpha-2 p / k+q+1, r)(-\alpha-2 p / k, 2 r) r !} \\
= & \sum_{p, q}(-)^{k q} \frac{(-p, k q) x^{p} y^{p-k q}}{(-\alpha-2 p / k+1, q) p ! q !} \cdot \frac{(-\alpha-2 p / k+1, q)(0, q)}{\left[\left(\frac{1}{2}\{-\alpha-2 p / k+1\}, q\right)\right]^{2}} \\
= & \sum_{p} \frac{x^{p} y^{p}}{p !}=e^{x y} \cdot{ }^{6}
\end{aligned}
$$

Thus $B_{n}$ and $L_{n}$ fulfill the definition of associate functions.

6 W. N. Bailey, Generalized Hypergeometric Series, Cambridge, 1935, p. 25. 
The Neumann series of the first kind gives expansions in

$$
\begin{aligned}
\left(\frac{1}{2} x\right)^{-v} J_{v+n}(x) & =\sum_{r}(-)^{r} \frac{\left(\frac{1}{2} x\right)^{n+2 r}}{\Gamma(v+n+r+1) r !} \\
& =\frac{1}{\Gamma(v+n+1)} \sum_{r}(-)^{r} \frac{\left(\frac{1}{2} x\right)^{n+2 r}}{(v+n+1, r) r !} \\
& =\frac{(-i)^{n}}{\Gamma(v+n+1)} B_{n}^{2}\left(v ; \frac{1}{2} i x\right) .
\end{aligned}
$$

The associate function is then

(i) $\Gamma(v+n+1) L_{n}^{2}(v,-2 i x)$

$$
\begin{aligned}
& =i^{n} \Gamma(v+n+1) \sum_{s} \frac{(-n, 2 s)(-2 i)^{n-2 s} y^{n-2 s}}{(-v-n+1, s)(1, s)} \\
& =\Gamma(v+n+1) \sum_{s}(-)^{s} \frac{(-n, 2 s) 2^{n-2 s}}{(-v-n+1, s) s !} y^{n-2 s}
\end{aligned}
$$

so that

$$
\begin{aligned}
f(x) & =\sum_{n} a_{n} \frac{x^{n}}{n !} \\
& =\sum_{n}\left(\frac{1}{2} x\right)^{-v} J_{v+n}(x) \cdot \frac{\Gamma(v+n+1)}{n !} \sum_{s}(-)^{s} \frac{(-n, 2 s) 2^{n-2 s}}{(-v-n+1, s) s !} a_{n-2 s .}
\end{aligned}
$$

The Jacobi polynomial may be similarly considered. The general form is

$$
\begin{aligned}
{ }_{2} F_{1}(-n, \alpha+n ; \gamma ; x)= & \sum_{r} \frac{(-n, r)(\alpha+n, r)}{(\gamma, r)(1, r)} x^{r} \\
= & \sum_{r}(-)^{n} \frac{(-n, r)(\alpha+n, n-r)}{(\gamma, n-r)(1, r)} x^{n-r} \\
= & (-)^{n}(\alpha+n, n) \\
& \cdot \sum_{r}(-)^{r} \frac{(-n, r) x^{n-r}}{(-\alpha-2 n+1, r)(\gamma, n-r) r !} \\
& =(-)^{n}(\alpha+n, n) L_{n}^{1}(\alpha, c x), \quad c_{h}=1 /(\gamma, h),
\end{aligned}
$$

so that according to Theorem II the associate function is

$$
\frac{(-)^{n}}{(\alpha+n, n)} B_{n}{ }^{1}(\alpha, y / c)=\frac{(-)^{n}}{(\alpha+n, n)} \sum_{s} \frac{(\gamma, n+s) y^{n+s}}{(\alpha+2 n+1, s) s !} .
$$


The Gegenbauer function, $C_{n}^{v}(x)$, includes the Legendre polynomial as the special case $v=\frac{1}{2}$. By definition, $\left(1-2 h x+h^{2}\right)^{-v}=\sum_{n} h^{n} \cdot C_{n}^{v}(x)$. Hence

$$
C_{n}^{v}(x)=\frac{(v, n)}{(1, n)} \sum_{s} \frac{(-n, 2 s)(2 x)^{n-2 s}}{(-v-n+1, s) s !}=\frac{(v, n)}{(1, n)} L_{n}^{2}(v, 2 x) .
$$

The associate function is

$$
\frac{(1, n)}{(v, n)} B_{n}^{2}\left(v, \frac{1}{2} y\right)=\frac{(1, n)}{(v, n)} \sum_{r} \frac{\left(\frac{1}{2} y\right)^{n+2 r}}{(v+n+1, r) r !}
$$

and

$$
f(x)=\sum_{n} a_{n} \frac{x^{n}}{n !}=\sum_{n} \frac{C_{n}^{v}(x)}{(v, n)} \sum_{r} \frac{a_{n+2 r}}{2^{n+2 r}(v+n+1, r) r !} .
$$

The Hermite polynomial

$$
H_{n}(x)=\sum_{s} \frac{(-n, 2 s)}{(1, s)} \frac{x^{n-2 s}}{2^{s}}=H_{n}^{2}(x)
$$

has the associate function $N_{n}^{2}(y)=\sum_{r} y^{n+2 r} / 2^{r}(1, r)$, so that

$$
f(x)=\sum_{n} a_{n} \frac{x^{n}}{n !}=\sum_{n} \frac{H_{n}(x)}{(1, n)} \sum_{r} \frac{a_{n+2 r}}{2^{r}(1, r)} .
$$

The Bessel functions of the third order introduced by P. Humbert ${ }^{7}$ provide a direct extension of the Neumann expansions. By definition, $\left(\frac{1}{3} x\right)^{-\alpha-\beta} J_{\alpha+2 n / 3, \beta+n / 3}(x)$

$$
\begin{aligned}
= & \frac{1}{\Gamma\left(\alpha+\frac{2}{3} n+1\right) \Gamma\left(\beta+\frac{1}{3} n+1\right)} \\
& \cdot \sum_{r}(-)^{r} \frac{\left(\frac{1}{3} x\right)^{n+3 r}}{\left(\alpha+\frac{2}{3} n+1, r\right)\left(\beta+\frac{1}{3} n+1, r\right) r !} \\
= & \frac{1}{\Gamma\left(\alpha+\frac{2}{3} n+1\right)} \sum_{r}(-)^{r} \frac{\left(\frac{1}{3} x\right)^{n+3 r}}{\Gamma\left(\beta+\frac{1}{3}(n+3 r)+1\right)\left(\alpha+\frac{2}{3} n+1, r\right) r !} \\
= & \frac{e^{-n \pi i / 3}}{\Gamma\left(\alpha+\frac{2}{3} n+1\right)} B_{n}^{3}(\alpha, c x), \quad c_{h}=\frac{\left(\frac{1}{3} e^{\pi i / 3}\right)^{h}}{\Gamma\left(\beta+\frac{1}{3} h+1\right)} .
\end{aligned}
$$

${ }^{7}$ P. Humbert, Comptes Rendus de l'Académie des Sciences, Paris, vol. 190 (1930), p. 59. 
Hence the associate function is

$$
\begin{aligned}
e^{n \pi i / 3} & \Gamma\left(\alpha+\frac{2}{3} n+1\right) L_{n}^{3}(\alpha, y / c) \\
= & e^{n \pi i / 3} \Gamma\left(\alpha+\frac{2}{3} n+1\right) \\
& \cdot \sum_{s}(-)^{s} \frac{(-n, 3 s) y^{n-3 s}}{\left(-\alpha-\frac{2}{3} n+1, s\right) s !} \frac{\Gamma\left(\beta+\frac{1}{3}(n+3 s)+1\right)}{\left(\frac{1}{3} e^{\pi i / 3}\right)^{n-3 s}} \\
= & \Gamma\left(\alpha+\frac{2}{3} n+1\right) \Gamma\left(\beta+\frac{1}{3} n+1\right) \\
& \cdot \sum_{s}(-)^{s} \frac{(-n, 3 s) y^{n-3 s}}{\left(-\alpha-\frac{2}{3} n+1, s\right)\left(-\beta-\frac{1}{3} n+1, s\right) s !} .
\end{aligned}
$$

The simplest two-variable expansions are those in products of onevariable functions. For example, the Neumann series of Bessel functions can be extended. Thus, if

$$
f(x, y)=\sum_{m, n} a_{m, n} \frac{x^{m}}{(1, m)} \frac{y^{n}}{(1, n)},
$$

then

$$
f(x, y)=\sum_{m, n} \alpha_{m, n} \frac{\left(\frac{1}{2} x\right)^{-v}\left(\frac{1}{2} y\right)^{-u}}{m ! n !} J_{v+m}(x) J_{u+n}(y),
$$

where

$$
\begin{aligned}
\alpha_{m, n}= & \Gamma(v+m+1) \Gamma(u+n+1) \\
& \cdot \sum_{r, s}(-)^{r+s} \frac{(-m, 2 r)(-n, 2 s) 2^{n+m-2 r-2 s}}{(-v-m+1, r)(-u-n+1, s) m ! n !} a_{m-2 r, n-2 s .}
\end{aligned}
$$

We have here made use of the associate functions for the Bessel functions as mentioned above.

There are expansions in mixed products. Thus, for $f(x, y)$ above, we have

$$
f(x, y)=\sum_{m, n} \frac{\beta_{m, n}}{m ! n !} H_{m}(x) C_{n}^{v}(y)
$$

where

$$
\beta_{m, n}=\frac{(1, n)}{(v, n)} \sum_{r, s} \frac{a_{m+2 r, n+2 s}}{2^{n+r+2 s}(v+n+1, s) r ! s !},
$$

giving the expansion in products of Hermite and Gegenbauer polynomials. 
P. Humbert ${ }^{8}$ introduced the set of confluent hypergeometric functions of two variables, special cases of which can be variously interpreted as extensions of the Sonine and Jacobi polynomials as well as the Bessel functions. Many of these provide expansions for two-variable functions in accordance with our theory. One of the simplest will illustrate. The polynomial

$$
\begin{aligned}
\phi_{2}\left(-m,-n ; \gamma ; x_{1}, x_{2}\right) & =\sum_{r, s} \frac{(-m, v)(-n, s)}{(\gamma, r+s) r ! s !} x_{1}^{r} x_{2}^{s}, \\
& =(-)^{m+n} H_{m}^{1}\left(c^{\prime} x_{1}\right) H_{n}^{1}\left(c^{\prime \prime} x_{2}\right), \\
& c^{\prime r} c^{\prime \prime s}=1 /(\gamma, r+s),
\end{aligned}
$$

has the associate function

$$
\begin{aligned}
(-)^{m+n} N_{m}^{1}\left(\frac{y_{1}}{c^{\prime}}, \frac{y_{2}}{c^{\prime \prime}}\right) & =\sum_{r, s}(-)^{m+n} \frac{y_{1}^{m+r} y_{2}^{n+s}}{c^{\prime m+r} c^{\prime \prime n+s} r ! s !} \\
& =\sum_{r, s}(-)^{m+n} \frac{(\gamma, r+s+m+n)}{(1, r)(1, s)} y_{1}^{m+r} y_{2}^{n+s}
\end{aligned}
$$

so that

$$
\begin{aligned}
f\left(x_{1}, x_{2}\right)= & \sum_{m, n} a_{m, n} \frac{x_{1}^{m} x_{2}^{n}}{m ! n !} \\
= & \sum_{m, n}(-)^{m+n} \frac{\phi_{2}\left(-m,-n ; \gamma ; x_{1}, x_{2}\right)}{(1, m)(1, n)} \\
& \cdot \sum_{r, s} \frac{(\gamma, r+s+m+n)}{(1, r)(1, s)} a_{m+r, n+8 .}
\end{aligned}
$$

In addition to expansions derived from the two basic sets above there are many expansions in functions whose associate functions may be obtained by other means. As one example of this, we mention the two-variable Hermite polynomials ${ }^{9} H_{m, n}\left(x_{1}, x_{2}\right)$ defined by the expansion

$$
\begin{aligned}
\exp \left\{h\left(a x_{1}+b x_{2}\right)+k\left(b x_{1}+c x_{2}\right)-\frac{1}{2}\left(a h^{2}+\right.\right. & \left.\left.2 b h k+c k^{2}\right)\right\} \\
& =\sum_{m, n} \frac{h^{m} k^{n}}{m ! n !} H_{m, n}\left(x_{1}, x_{2}\right) .
\end{aligned}
$$
p. 73.

${ }^{8}$ P. Humbert, Proceedings of the Royal Society of Edinburgh, vol. 41 (1921),

${ }^{9}$ Ch. Hermite, Oeuvres, vol. 2, pp. 293-308. 
If we write $h a+k b=y_{1}, h b+k c=y_{2}$, then

$$
e^{x_{1} y_{1}+x_{2} y_{2}}=\sum_{m, n} \frac{H_{m, n}\left(x_{1}, x_{2}\right)}{m ! n !} h^{m} k^{n} \exp \left\{\frac{1}{2}\left(a h^{2}+2 b h k+c k^{2}\right)\right\},
$$

so that the function associate to $H_{m, n}\left(x_{1}, x_{2}\right)$ is

$$
\begin{aligned}
N_{m, n}\left(y_{1}, y_{2}\right)= & \frac{\left(c y_{1}-b y_{2}\right)^{m}\left(-b y_{1}+a y_{2}\right)^{n}}{\Delta^{m+n}} \\
& \cdot \exp \left\{\frac{c y_{1}^{2}+2 b y_{1} y_{2}+a y_{2}^{2}}{2 \Delta}\right\}
\end{aligned}
$$

where $\Delta=a c-b^{2} \neq 0$.

Restrictions of space prevent our continuing with the almost endless variety of illustrations of the theory. These methods can be particularly useful in obtaining the explicit forms of new expansions. The detailed properties of the sets of functions will suffice to answer questions of convergence.

For the original suggestion of this work and continued counsel, the author wishes to express his appreciation to Harry Bateman and to E. T. Bell.

Queens College 